\title{
Integrated Photonics for Wireless and Satellite Applications
}

\author{
Katarzyna Balakier $^{1 *}$, Ahmad W. Mohammad ${ }^{1 * *}$, Martyn Fice $^{1}$, Guillermo Carpintero ${ }^{2}$, Frederic van Dijk ${ }^{3}$, \\ Alwyn J. Seeds ${ }^{1}$ and Cyril C. Renaud ${ }^{1}$ \\ ${ }^{1}$ University College London, Torrington Place, London, WC1E 7JE, UK \\ ${ }^{2}$ Universidad Carlos III de Madrid, Avenida de la Universidad 30, 28911 Leganes, Madrid, Spain \\ ${ }^{3}$ III-V Lab, Campus de Polytechnique, 91767 Palaiseau Cedex, France \\ ** now with LioniX International BV \\ *k.balakier@ucl.ac.uk
}

\begin{abstract}
The concept of using Photonic Integrated Circuits for generation of tunable mm-wave signals for wireless and satellite communication application is presented. The paper outlines the requirements for frequency stabilization and power consumption of semiconductor lasers when implemented in terrestrial wireless and satellite communication applications.

(C) 2020 The Author(s)
\end{abstract}

\section{Introduction}

In recent years there could be observed a continuous increase in demand for cellular data as well as other mobile services, which in turn has resulted in an exponential increase in network capacity demand. The roll-out and expansion of $5 \mathrm{G}$ networks illustrates the future need for systems operating at $\mathrm{Q}, \mathrm{V}$ and $\mathrm{W}$ bands. It is at these higher frequency regions where photonics technology can offer a particular advantage over the electronics and RF solutions. This is due to broader bandwidth and frequency tunability of photonic components as well as utilisation of optical heterodyne techniques for high frequency signal generation, modulation and frequency conversion. Photonics integration is key to successful deployment of photonics solutions for a broader range of both terrestrial wireless and satellite communication applications.

This paper summarises experimental demonstrations performed using a highly integrated PIC showing transmitter and receiver functionality. We also propose the use of this highly universal PIC as a transceiver, offering generation, detection and frequency down-/up-conversion of signals in a satellite communication link.

\section{Photonic integration}

The PIC, presented for the first time in 2014 [1], was an outcome of the iPHOS collaborative project with the aim of demonstrating the capability to monolithically integrate DFB lasers, SOAs and broad bandwidth photodiodes on a single InP chip for different applications requiring $>60-\mathrm{GHz}$ technology. Fig. 1 shows the PIC and its potential implementation as a microwave photonics transceiver.

\section{Satellite applications}

The developments towards integration of satellite communication into the mainstream communication networks particularly in the context of $5 \mathrm{G}$ - create an opportunity to consider various solutions for future satellite systems. The alignment of standards and technology across terrestrial wireless and satellite networks would have a positive impact on the integrated photonics technology by increasing demand for higher volume and thus lowering the cost. When discussing photonics for space application, it is important to consider stringent frequency stability requirements for the satellite communication systems. Satellite transmission bands are in the $\mathrm{GHz}$ range while channels widths can be sub-MHz. The out of band signal suppression should remain below $40 \mathrm{~dB}$ (bandwidth dependent). This is difficult to achieve for free running lasers due to temperature drift of about $14 \mathrm{GHz} /{ }^{\circ} \mathrm{C}$. It means that a heterodyne signal generated by free-running lasers would drift out of band with the $0.07^{\circ} \mathrm{C}$ temperature change. The problem is further exacerbated by the equipment temperature specification requirement of constant performance across $60^{\circ} \mathrm{C}$ temperature range. Narrow linewidth lasers with ultra-sensitive temperature control or laser phase locking techniques [2], [3] can offer solutions to this problem.

The majority of satellites use solar panels to generate the necessary electrical power for their equipment. The total power available for payload equipment is often limited to only a few kilowatts (depending on the platform size, orbit and mission type). This makes DC power a very limited resource. At the same time efficiency $\left(\mathrm{P}_{\text {opt }} / \mathrm{P}_{\mathrm{DC}}\right)$ of a semiconductor laser is typically around $20 \%$ (temperature depended), and decrease further as the temperature 
increases. It creates a need for a thermoelectric cooler (TEC), which further increases equipment power consumption.

Photonic integration has already offered a solution to considerably reduce optical coupling losses between what otherwise would be discrete components. In this paper we describe an approach to using the same lasers for the transmitter and the receiver unit to further reduce the overall power consumption of photonics-based systems. In the transmit path the two laser tones are combined using a 2 × 2 MMI coupler, data is modulated on the optical carriers before the signal is converted to the RF domain by a corresponding UTC-PD connected to an antenna [4]. In the receive path, the incoming RF signal needs to be down-converted to IF frequency before it can be recovered for data processing. We have investigated two approaches to signal down-conversion. Firstly, the integrated UTCPD was used as an optically pumped mixer for down-conversion of a received $70 \mathrm{GHz}$ signal to intermediate frequency (IF) that was tuneable from $0.5 \mathrm{GHz}$ to $16.4 \mathrm{GHz}$ [5]. The $5 \mathrm{Gbps}$ at $35 \mathrm{GHz}$ carrier was also demonstrated, although a UTC-PD without integrated lasers was used in this experiment [6]. The frequency of the IF signal is defined by the wavelength spacing between two DFB lasers, and is tuneable from $5 \mathrm{GHz}$ to $120 \mathrm{GHz}$. The alternative approach to down-conversion was to use an external RF mixer (with lower conversion loss), which was driven with an electrical LO signal generated by a UTC-PD integrated with two DFB lasers [7]. Fig. 2 shows eye diagrams generated by using a real-time captured signal of various transmission experiments implementing the PIC and UTC-PD as a transmitter or receiver.
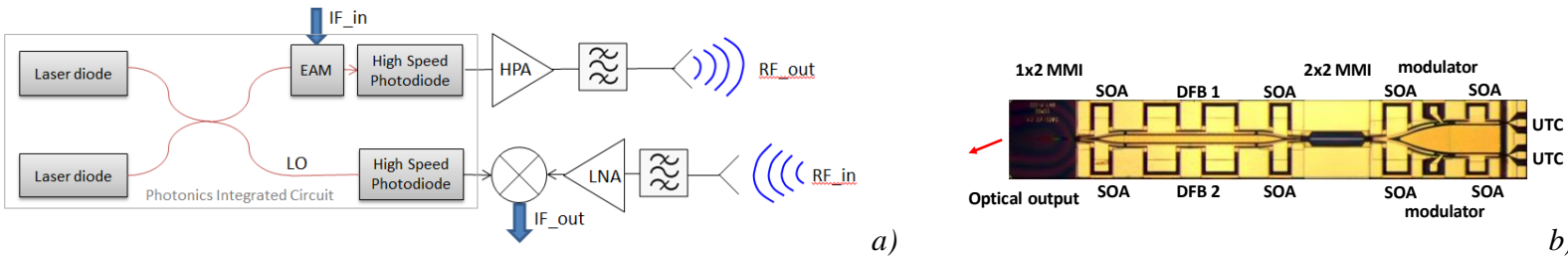

a)

Fig. 1. (a) Schematic of a transceiver on PIC, (b) photograph of the InP chip used in the first experiments

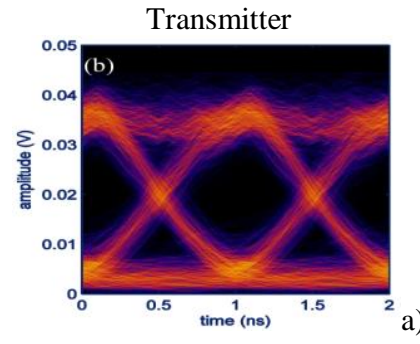

1 Gbps at $105.4 \mathrm{GHz}[4]$
Receiver with Optically pumped mixer

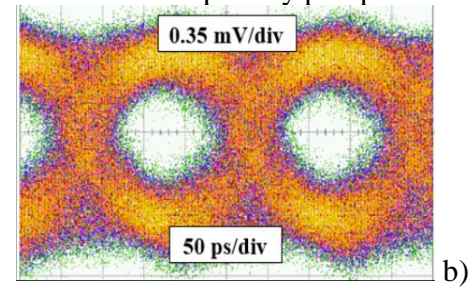

5 Gbps at $35 \mathrm{GHz}[6]$

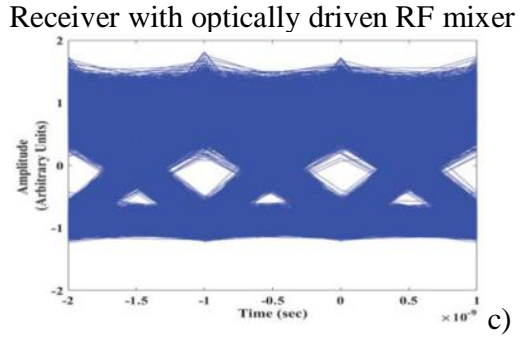

$1 \mathrm{Gbps}$ OOK at $64.5 \mathrm{GHz}$ [7]

Fig. 2. Eye diagram of the recovered signal for links implementing PIC and UTC-PD as transmitter a) and receiver $b$ ) and c)

In conclusion, the use of the same lasers for both transmit and receive functionality has a potential to reduce the overall DC power consumption of the system. However, using the same laser for up-converting the transmit signal and down-converting the received signal will require system level frequency planning. Although the emphasis of this paper is on converged implementation of photonics by terrestrial and satellite telecommunications networks, PICs capable of generating broadly tunable $\mathrm{mm}$-wave and $\mathrm{THz}$ signals can be considered for other space-based applications, such as satellite Earth observation and deep space scientific missions.

We acknowledge the support of EPSRC UKRI through "Photonic Systems for next generation satellites" project (EP/S000976/1) and the European Commission through iPHOS (257539) and FiWiN5G (642355) projects.

[1] F. van Dijk, et al., "Photonic integrated circuit on InP for millimeter wave generation," SPIE 8988, 89880Q (2014).

[2] K. Balakier, et al., "Integrated Semiconductor Laser Optical Phase Lock Loops [Invited Paper]," J. Sel. Top. Quantum Electron. 24, (2018).

[3] K. Balakier, et al., "Optical injection locking of monolithically integrated photonic source for generation of high purity signals above 100 GHz," Opt. Express 22, 29404 (2014).

[4] Z. Yang et al.,"A 1 Gbps $105.4 \mathrm{GHz}$ link with a directly modulated photonic integrated dual laser source," in Conference on Lasers and Electro-Optics (CLEO), 2014,

[5] A. W. Mohammad, et al., "Optically Pumped Mixing in Photonically Integrated Uni-Travelling Carrier Photodiode," Int. Conf. Infrared, Millimeter, Terahertz Waves, IRMMW-THz (2018).

[6] A. W. Mohammad, et al.,"5 Gbps Wireless Transmission Link with an Optically Pumped Uni-Traveling Carrier Photodiode Mixer at the Receiver," Opt. Express, vol. 26, N, 2018.

[7] A. W. Mohammad, et al., "60 GHz Wireless Link Implementing an Electronic Mixer Driven by a Photonically Integrated Uni-Traveling Carrier Photodiode at the Receiver," in IEEE Microwave Photonics 761579 (2018). 